\title{
Early Time-Dependent Dynamic Changes of TBET and GATA3 mRNA Expressions in Patients with Acute Coronary Syndrome
}

\author{
Timothy H. Rainer, ${ }^{1}$ Colin A. Graham, ${ }^{1}$ Rebecca W. Y. Chan, ${ }^{2}$ Cangel P. Y. Chan, ${ }^{1}$ \\ Patrick C. F. Tan, ${ }^{1}$ Gabriel W. K. Yip, ${ }^{3}$ and Cheuk-Man $\mathbf{Y u}^{3}$ \\ ${ }^{1}$ Accident and Emergency Medicine Academic Unit, The Chinese University of Hong Kong, Prince of Wales Hospital, Hong Kong \\ ${ }^{2}$ Department of Chemical Pathology, Li Ka Shing Institute of Health Sciences, The Chinese University of Hong Kong, \\ Prince of Wales Hospital, Hong Kong \\ ${ }^{3}$ Division of Cardiology, Department of Medicine \& Therapeutics, The Chinese University of Hong Kong, \\ Prince of Wales Hospital, Hong Kong
}

Correspondence should be addressed to Timothy H. Rainer; thrainer@cuhk.edu.hk

Received 26 June 2013; Revised 6 August 2013; Accepted 17 August 2013

Academic Editor: Francisco Blanco-Vaca

Copyright (C) 2013 Timothy H. Rainer et al. This is an open access article distributed under the Creative Commons Attribution License, which permits unrestricted use, distribution, and reproduction in any medium, provided the original work is properly cited.

Background. T-box expressed in T cells (TBET) and guanine adenine thymine adenine sequence-binding protein 3 (GATA3) play important roles in the differentiation of Th1 and Th2 subsets, which contributes to the progression of acute coronary syndrome (ACS). Objective. This study aimed to investigate the temporal change of TBET/GATA3 mRNA ratio in ACS. Methods. Thirtythree patients suspected of ACS with symptom onset within 24 hours were recruited. Blood samples were taken after arrival at the emergency department and at hourly intervals until the 6th hour. The mRNA expressions of TBET and GATA3 were quantified by a real-time RT-qPCR. Results. The TBET/GATA3 mRNA ratio was elevated dramatically in patients with acute myocardial infarction (AMI) and exhibited biphasic M-shaped release kinetics with two distinct peaks. The ratio was elevated 2 hours after symptom onset, dropped to the lowest level at 10 hours, and rose to the second peak at 14 hours. A similar biphasic M-shaped curve was observed in AMI patients with blood samples taken prior to any intervention. Conclusions. The TBET/GATA3 mRNA ratio was elevated in AMI patients throughout most of the first 20 hours after symptom onset. The biphasic M-shaped release kinetics was more likely to reflect pathophysiological changes rather than treatment effects.

\section{Introduction}

On activation, $\mathrm{T}$ lymphocytes differentiate into T-helper (Th) 1 and Th2 subsets, and although the control of the Th1/Th2 imbalance is not fully elucidated, there is growing evidence to suggest that two transcription factors, T-box expressed in $\mathrm{T}$ cells (TBET) and guanine adenine thymine adenine sequence-binding protein 3 (GATA3), play important roles in such differentiation [1-4].

Th1 is essential in the process of plaque instability and plaque rupture, which in turn are common features in the pathogenesis of acute coronary syndrome (ACS) [5-7]. Upregulation of Th1 response has been demonstrated in the circulating lymphocytes of patients with ACS $[6,8]$. In contrast, $\mathrm{Th} 2$ has rarely been shown in atherosclerotic lesions. Recent studies suggest that loss of Th1 and Th2 balance contributes to plaque rupture and the onset of ACS $[6,9]$. Th1 and Th2 imbalances in the pathogenesis of ACS have been observed coherently from transcription to protein levels in both animal models and human subjects [8-13]. The relative expression of TBET and GATA3, resulting in a swing in the Th1/Th2 pendulum, has been implicated in several immunological diseases and may provide better prognostic and diagnostic information than downstream cytokines.

In our recent study, we have demonstrated that the TBET/ GATA3 mRNA ratio was elevated in patients with ACS and associated with disease severity [14]. However, little is known about whether the biological and temporal changes in circulating leukocyte TBET/GATA3 mRNA balance are associated 
with the ACS progress and whether the changes reflect the development of the pathophysiological processes or the effect of therapeutic intervention. This study aimed to investigate the temporal dynamic changes in initial mRNA expressions of TBET/GATA3 ratio in patients presenting with various clinical stages of coronary artery diseases including stable angina (SA), unstable angina (UA), and acute myocardial infarction (AMI).

\section{Materials and Methods}

2.1. Study Design and Setting. This prospective observational study was conducted at the Emergency Department (ED) of the Prince of Wales Hospital in Hong Kong. Ethical approval was obtained from the local Institutional Review Board, and written consent was obtained from all patients or the closest available relative. Patients were recruited consecutively between 9:00 a.m. and 5:00 p.m. from Monday to Friday. Ageand sex-matched healthy subjects, which acted as a healthy control group, had no history of chronic or recent acute illness. Both the research nurse who collected clinical data and the scientist who performed the laboratory analysis were blinded to the clinical outcome.

2.2. Inclusion and Exclusion Criteria. Patients aged $\geq 18$ years presenting to the $\mathrm{ED}$ with nontraumatic chest or epigastric pain of less than 24 hours since acute symptom onset, and with a provisional diagnosis of ACS were recruited into the study. Patients were excluded if they had sustained an AMI within 10 days prior to arrival, had received an intramuscular injection or sustained trauma within 3 days, had renal impairment, severe muscular or infectious disease, asthma, systemic lupus erythematosus, or obvious noncardiac chest pain (e.g., pneumothorax, rib fractures, pneumonia, or trauma).

2.3. Diagnosis and Treatment. Patients were diagnosed as SA (a disease control group) if there was a typical chest pain occurring with activity but relieved by rest or vasodilators within 10 minutes of onset. ACS patients were diagnosed according to the published consensus of the European Society of Cardiology and American College of Cardiology Committee at the time of the study $[15,16]$. Patients were diagnosed as (i) UA patient (a disease control group) if there was an accelerating or "crescendo" pattern of chest pain which either occurred at rest or lasted longer than 10 minutes or was not responsive to medication; (ii) AMI patient if there was an increase in cardiac troponin $\mathrm{T}$ (cTnT) with at least one value above $0.06 \mu \mathrm{g} / \mathrm{L}$ together with evidence of myocardial ischemia with at least one of the following: (a) symptoms of ischemia (chest pain); (b) electrocardiogram (ECG) changes indicative of new ischemia (new ST-T changes or new left branch bundle block (LBBB) or T wave inversion); (c) development of pathological Q wave changes in ECG; (d) echo evidence of new loss of viable myocardium or new regional wall motion abnormality [16]. AMI patients were further subdivided into patients with ST elevation myocardial infarction (STEMI) and non-ST elevation MI (NSTEMI). Patients were managed according to the standardized treatment protocols
$[15,17]$ and treated with aspirin, nitrates, enoxaparin, thrombolysis, and/or coronary angiography, and percutaneous coronary intervention (PCI) as clinically indicated.

2.4. Blood Collection, Processing, and Storage. A $12 \mathrm{~mL}$ of venous blood sample was withdrawn from each patient as soon as possible after arrival at the ED $\left(T_{0}\right)$ and at hourly intervals until the 6th hour $\left(T_{1}, T_{2}, T_{3}, T_{4}, T_{5}\right.$, and $\left.T_{6}\right)$ and then immediately transferred into EDTA tubes. A venous blood sample was also withdrawn from each control subject at $T_{0}, T_{3}$, and $T_{6}$ after written consent was obtained.

After centrifugation at $1600 \mathrm{~g}$ for 10 minutes at $4^{\circ} \mathrm{C}$, the buffy coat layer was transferred to a microcentrifuge tube and further centrifuged at $230 \mathrm{~g}$ for 5 minutes at $4^{\circ} \mathrm{C}$ to remove the plasma. Then, the buffy coat was removed, lysed, and preserved in lysis buffer (Trizol LS Reagent, Invitrogen). The total RNA in the samples was extracted by the RNeasy Mini Kit (Qiagen Inc., Canada) following manufacturer's instructions. All RNA samples were pretreated with Amplification Grade Deoxyribonuclease I (Invitrogen) and then stored at $-80^{\circ} \mathrm{C}$ until further processing. Blood samples were also collected for routine measurement of $c \mathrm{TnT}$ and other biochemical parameters. The upper reference limit for $\mathrm{c} T n \mathrm{~T}$ was $0.03 \mu \mathrm{g} / \mathrm{L}$.

2.5. Real-Time Quantitative Reverse Transcription Polymerase Chain Reaction (RT-qPCR). A quantitative RT-qPCR by the ABI Prism 7700 Sequence Detector System (Applied Biosystems, Foster City, CA, USA) was used to determine the mRNA expressions of TBET, GATA3, and glyceraldehyde 3-phosphate dehydrogenase (GAPDH) which was used as a housekeeping gene for normalization. We have developed RT-qPCR assays for TBET (Gene ID: 30009) and GATA3 (Gene ID: 2625). The assay for TBET spans exons 1 and 2 of the transcript located on $17 \mathrm{q} 21.32$ and the assay for GATA3 spans exons 2 and exon 3 of the transcript located on 10p15. Both assays are intron-spanning. The primer and probe sequences of TBET, GATA3, and GAPDH were designed using Primer Express (v2.0) and are summarized in Supplemental Table 1 (see Supplementary Material available online at http://dx.doi.org/10.1155/2013/139895). The RTqPCR amplifications were performed in a volume of $25 \mu \mathrm{L}$ at $50^{\circ} \mathrm{C}$ for 2 minutes, $60^{\circ} \mathrm{C}$ for 30 minutes, and $95^{\circ} \mathrm{C}$ for 5 minutes followed by 40 cycles of $94^{\circ} \mathrm{C}$ for 20 seconds and $60^{\circ} \mathrm{C}$ for 1 minute. Each sample was run in duplicate. The results of RT-qPCR were analyzed by the Sequence Detection Software version 1.9 (Applied Biosystems, Foster City, CA, USA). Calibration curves for TBET, GATA3 and GAPDH mRNA measurements were prepared by serial dilutions of HPLC-purified single-stranded synthetic oligonucleotides. The concentrations of TBET and GATA3 mRNA were expressed in copies/pg of GADPH mRNA.

2.6. Statistical Analysis. Statistical analysis was performed using MedCalc version 11.5.1 (Mariakerke, Belgium) and Statview for Windows version 5.0 Statistical Analysis Software (Abacus Concepts, SAS Institute, Cary, NC, USA). Categorical variables were compared using Chi-square test. Continuous variables were compared by using Mann-Whitney 
TABLE 1: Baseline characteristics of AMI and non-AMI patients.

\begin{tabular}{|c|c|c|c|c|}
\hline & Total $(n=33)$ & Non-AMI $(n=19)$ & AMI $(n=14)$ & $P$ Value \\
\hline \multicolumn{5}{|l|}{ Characteristic } \\
\hline Age-median years (IQR) & $73(56-81)$ & $74(55-83)$ & $65(56-81)$ & 0.477 \\
\hline Male gender-no. (\%) & $20(58)$ & $10(53)$ & $9(64)$ & 0.754 \\
\hline $\begin{array}{l}\text { Time from symptom onset to blood taking for } \\
\text { measurements of TBET \& GATA3 - median hours (IQR) }\end{array}$ & $4.0(3.0-8.9)$ & $3.5(2.6-5.8)$ & $5.3(4.0-12.0)$ & 0.068 \\
\hline \multicolumn{5}{|l|}{ Past history-no. (\%) } \\
\hline Myocardial infarction & $5(15)$ & $2(11)$ & $2(14)$ & 0.832 \\
\hline Cerebrovascular disease & $7(21)$ & $4(21)$ & $3(21)$ & 0.686 \\
\hline Heart failure & $6(18)$ & $4(21)$ & $1(7.0)$ & 0.542 \\
\hline Diabetes & $11(33)$ & $4(21)$ & $6(43)$ & 0.335 \\
\hline Hypertension & $21(64)$ & $11(58)$ & $9(64)$ & 0.991 \\
\hline Current smoker & $9(27)$ & $5(26)$ & $4(29)$ & 0.801 \\
\hline Ex-smoker & $3(24)$ & $0(0.0)$ & $2(14)$ & 0.336 \\
\hline Obesity & $6(18)$ & $1(5.0)$ & $4(29)$ & 0.176 \\
\hline Hyperlipidemia & $6(18)$ & $2(11)$ & $4(29)$ & 0.383 \\
\hline Atrial fibrillation & $4(12)$ & $3(16)$ & $1(7)$ & 0.832 \\
\hline \multicolumn{5}{|l|}{ Medications-no. (\%) } \\
\hline Warfarin & $2(6.0)$ & $2(5.9)$ & $0(0.0)$ & 0.607 \\
\hline Aspirin & $15(46)$ & $9(47)$ & $6(17.6)$ & 0.923 \\
\hline Beta-blocker & $9(33)$ & $4(21)$ & $4(11.8)$ & 0.931 \\
\hline \multicolumn{5}{|l|}{ Final diagnosis-no. (\%) } \\
\hline Stable angina & $8(24)$ & $8(42)$ & - & \\
\hline Unstable angina & $11(33)$ & $11(58)$ & - & \\
\hline NSTEMI & $5(15)$ & - & $5(36)$ & \\
\hline STEMI & $9(27)$ & - & $9(64)$ & \\
\hline Mortality-no. (\%) & $2(6.0)$ & $0(0.0)$ & $2(14)$ & 0.336 \\
\hline
\end{tabular}

$U$ test or unpaired $t$-test between the two groups, and by using ANOVA test followed by a post hoc Bonferroni test for multiple comparisons. A $P$ value of less than 0.05 was considered as statistically significant.

In this pragmatic study, it was important that essential therapies continued without delay or interruption. Inevitably, this meant that blood samples could not be taken at some time points. In order to proceed with statistical analysis, missing data points were filled using a statistical technique described by Snedecor and Cochran [18]. 16\% of the total data points were missing and were computed using the following formula:

$$
X=\frac{a T+b B-S}{(a-1)(b-1)},
$$

where $a$ is the number of patients, $b$ is the number of time points, $T$ is the sum of data points from the same patient with missing time point, $B$ is the sum of data points from the same time point as missing time point, and $S$ is the sum of all observed data points.

In order to determine whether the dynamic changes of the TBET/GATA3 mRNA ratio were related to the treatment effect or primarily to pathophysiological changes, the changes of the ratio in 136 patients (47 AMI and 89 non-AMI) with single blood samples taken at ED arrival were used for comparison [14].

\section{Results}

Between 12 September, 2007 and 12 February, 2008, 33 patients were recruited to the study with a provisional diagnosis of ACS. Baseline characteristics of the patients are shown in Table 1. The median age of the 28 healthy subjects acting as the healthy control group was 58 years (IQR 53-62), and there were 18 males (64.0\%). The 95th percentile for the TBET/GATA3 mRNA ratio was 1.26 in the control group. Figure 1 shows the temporal changes of the TBET/GATA3 mRNA ratio in patients with AMI and nonAMI over 20 hours from the symptom onset and in healthy control subjects. The TBET/GATA3 mRNA ratios in the AMI group were significantly higher than those in the non-AMI group which included patients with SA and UA acting as the disease control group at 6 hours (1.7 versus $1.1, P=0.007), 8$ hours (1.6 versus $1.2, P=0.006)$, 14 hours (2.8 versus $1.2, P=$ $0.035), 16$ hours $(2.4$ versus $1.2, P=0.020)$, and almost at 18 hours $(1.7$ versus $0.8, P=0.060)$. The TBET/GATA3 mRNA ratio exhibited biphasic $\mathrm{M}$-shaped release kinetics. The ratio was elevated 2 hours after the symptom onset, dropped to the lowest level at 10 hours, and rose to the second peak at 14 hours. The biphasic M-shaped curve was also observed by plotting the TBET/GATA3 mRNA ratio against the time from ED arrival (Figure 2). The temporal changes in the TBET/GATA3 mRNA ratio for each individual patient were 


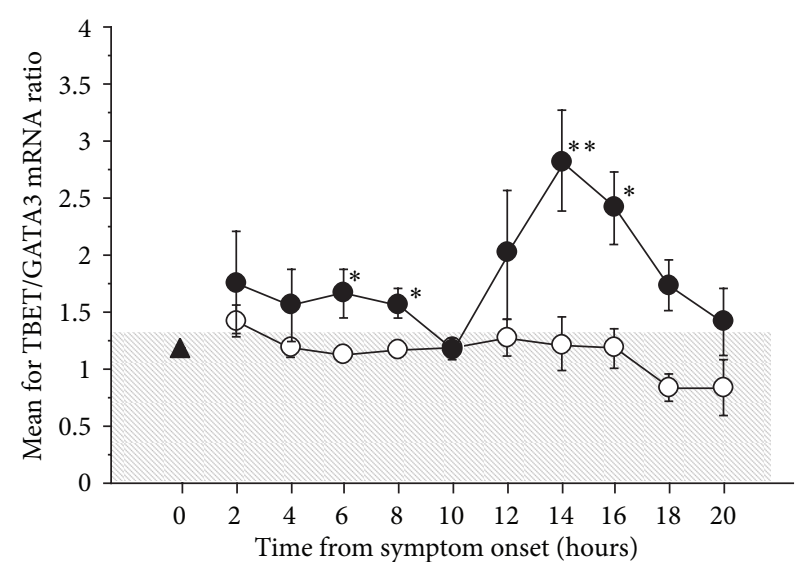

FIgURE 1: Temporal changes in the TBET/GATA3 mRNA ratio after symptom onset in 14 AMI patients $(\bullet), 19$ non-AMI patients (o), and 28 control subjects $(\boldsymbol{\Delta})$. Data is presented as mean \pm 1 SEM. The grey zone represents the ratio within the 95th percentile (i.e., $\leq 1.26$ ). Significant difference in TBET/GATA3 mRNA ratio was found between AMI and non-AMI patients with $P<0.05$ by using $t$-test $(*)$ or post hoc Bonferroni test $(* *)$.

shown in Figure 3. The ratios were above the 95th percentile in only 2 out of 8 SA patients (25.0\%) (Figure 3(a)) while there were 6 out of $11 \mathrm{UA}$ patients $(54.5 \%)$ with the ratios above the 95th percentile (Figure 3(b)). Among the 14 AMI patients, the ratios were above the 95th percentile in all the 9 STEMI patients (100.0\%) (Figure 3(c)) and all the 5 NSTEMI patients (100.0\%) (Figure 3(d)).

The areas below the curves for all the patient groups were significantly greater than that for the control group $(P<$ 0.0001 ). The area below the curve for the STEMI group was significantly greater than that for the SA group $(P=0.041)$ while for the NSTEMI was significantly greater than those for the SA $(P=0.001)$ and the UA $(P=0.008)$ groups.

Significant difference in TBET/GATA3 mRNA ratio at $T_{0}$ was found across different groups (Kruskal-Wallis Test, $P=$ 0.0006) (Figure 4). The median TBET/GATA3 mRNA ratio was highest in NSTEMI patients (1.97 (IQR: 1.54-3.08)), followed by STEMI (1.32 (IQR: 1.19-1.84)), UA (1.18 (IQR: 1.101.33)), and SA (1.05 (IQR: 0.92-1.11)) and lowest in healthy subjects (0.97 (IQR: 0.65-1.10)).

Based on Figure 1, it was difficult to determine whether the biphasic M-shaped release kinetics was due to treatment effects or pathophysiological mechanisms. In order to find out the underlying reason, it was necessary to investigate the temporal changes of TBET/GATA3 mRNA ratio in samples taken prior to any intervention. The first samples taken from the 33 patients exhibited similar release kinetics as shown in Figure 1. However, the sample size was too small for robust analysis. Therefore, blood samples taken from the 136 patients prior to any intervention recruited in our previous study were investigated [14]. A similar biphasic $M$-shaped curve as shown in Figure 5 was observed in the 47 AMI patients. Changes in the TBET/GATA3 mRNA ratio of the 89 non-AMI patients resembled those of the AMI patients. The TBET/GATA3 mRNA ratios in the AMI group

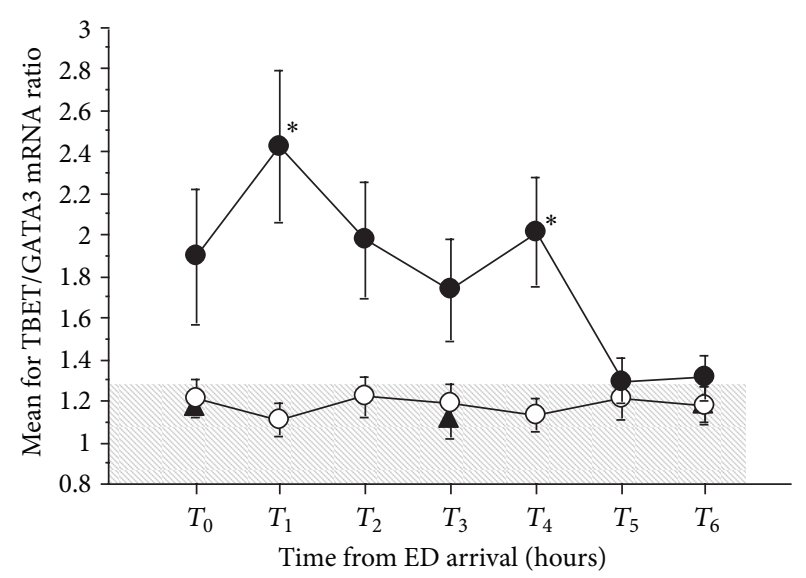

Figure 2: Temporal changes in the TBET/GATA3 mRNA ratio after ED arrival in 14 AMI patients (•), 19 non-AMI patients (O), and 28 control subjects $(\mathbf{\Delta})$. Data is presented as mean \pm 1 SEM. The grey zone represents the ratio within the 95th percentile (i.e., $\leq 1.26$ ). Significant difference in TBET/GATA3 mRNA ratio was found between AMI and non-AMI patients with $P<0.05$ by using $t$-test $(*)$.

were significantly higher than those in the non-AMI group at 2 hours (1.7 versus $0.9, P=0.001), 4$ hours (2.2 versus $1.1, P=0.003), 6$ hours (2.0 versus $1.0, P=0.0006), 8$ hours (2.2 versus 1.1, $P=0.0003$ ), 10 hours (1.9 versus 1.0 , $P=0.003), 12$ hours (1.8 versus $1.0, P=0.011), 14$ hours $(2.8$ versus $1.2, P=0.010), 16$ hours (1.7 versus $0.9, P=0.044)$, and 20 hours ( 1.8 versus $1.0, P=0.002$ ).

\section{Discussion}

The Evidence shows that the progression of atherosclerotic disease is associated with systemic immune activation [11,19]. Different stages of atherosclerotic disease are associated with distinct differentiation patterns of activated T-helper cells. Many studies have demonstrated that Th1/Th2 imbalance contributes to the initiation and progression of ACS $[6,9]$. Also, there is growing evidence to suggest that two upstream transcription factors, TBET and GATA3, governing T-helper cell differentiation, play an important role in the progression of atherosclerosis and its complications [20-23]. TBET promotes Th1 lineage commitment [24] and maintains the Th1 mediated immune response, which fosters macrophage activation, infiltration, and the resulting ACS [25]. Th1 cells and their related cytokines play a crucial role in inflammatory cell infiltration to the lipid plaque [9-12]. Th1 cytokines were elevated in ACS patients with higher concentrations in AMI patients compared with SA or UA patients [10]. GATA3 promotes Th2 differentiation [26] and induces Th2 cytokines production in an analogous way to TBET [27].

In our previous study [14], the diagnostic performances of initial CK, initial cTnT, GATA3 mRNA, TBET mRNA, and TBET/GATA3 mRNA ratio were compared using receiver operating characteristics (ROC) curve analysis. The area under the curve (AUC) for TBET/GATA3 mRNA ratio was 0.897 (95\% CI $0.833-0.943)$, which was greater than that of the other variables analyzed. There were significant 
Patient 11

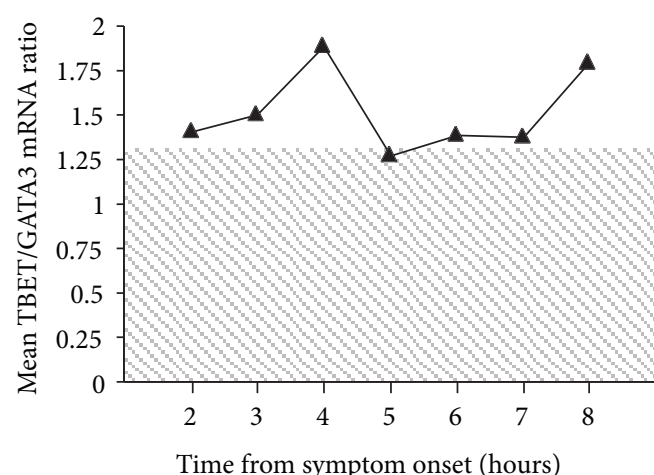

Patient 17



Time from symptom onset (hours)

Patient 27



Time from symptom onset (hours)

Patient 35

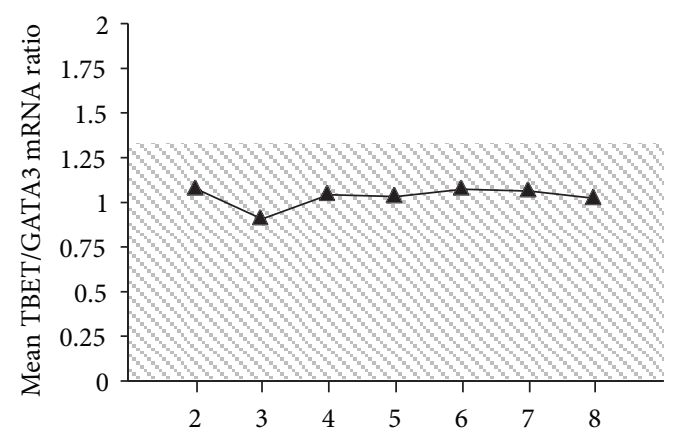

Time from symptom onset (hours)
Patient 12

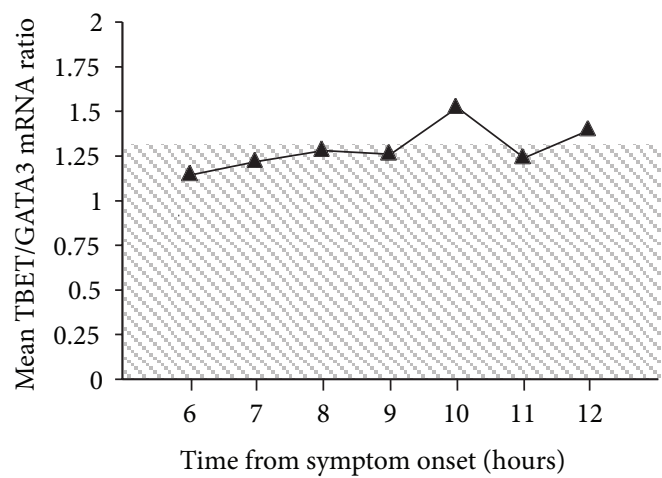

Patient 24

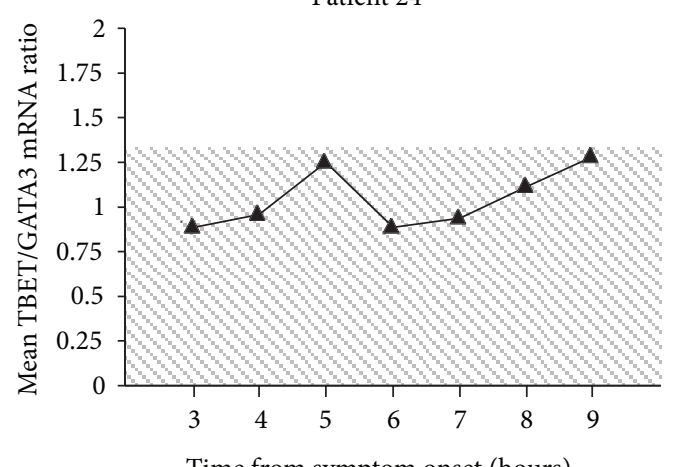

Time from symptom onset (hours)

Patient 31

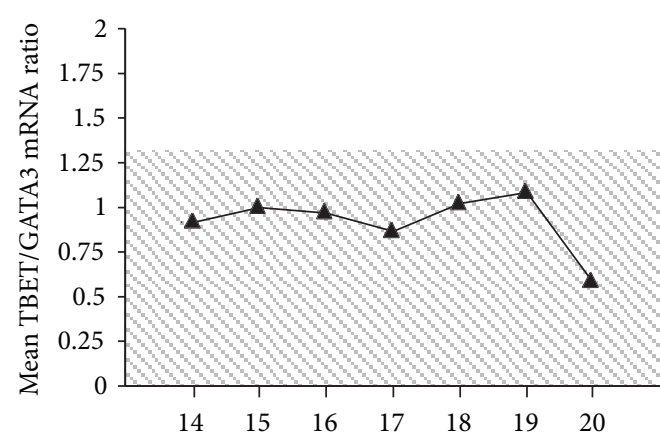

Time from symptom onset (hours)



Time from symptom onset (hours)

(a)

Figure 3: Continued. 
Patient 1



Time from symptom onset (hours)

Patient 14



Time from symptom onset (hours)

Patient 26

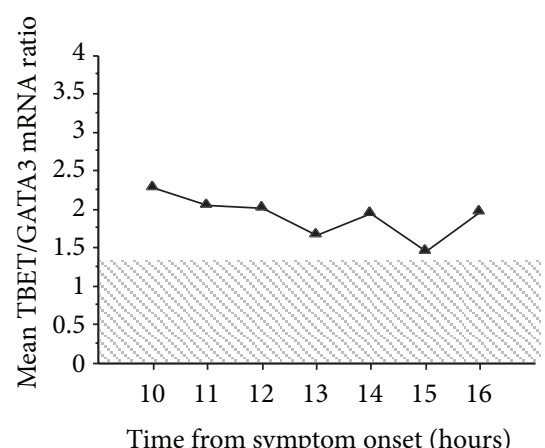

Patient 33

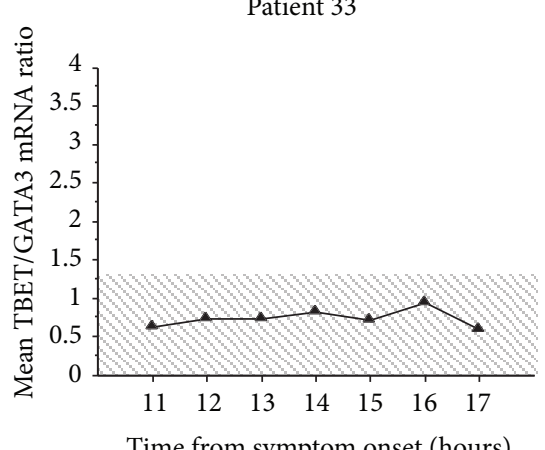

Time from symptom onset (hours)
Patient 5

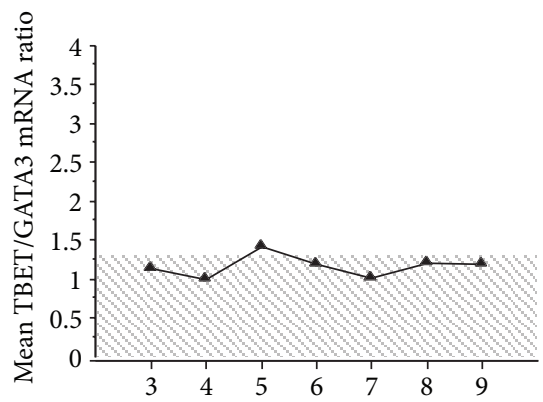

Time from symptom onset (hours)

Patient 16

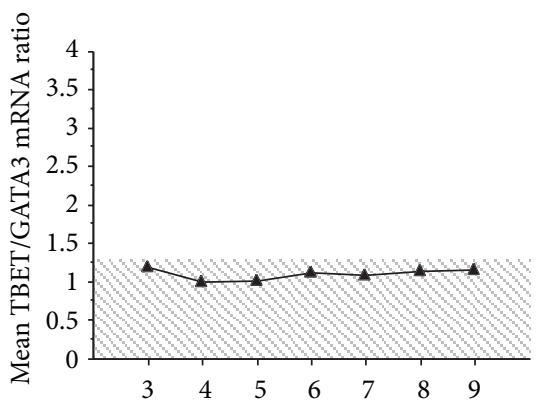

Time from symptom onset (hours)

Patient 30



Time from symptom onset (hours)

Patient 40

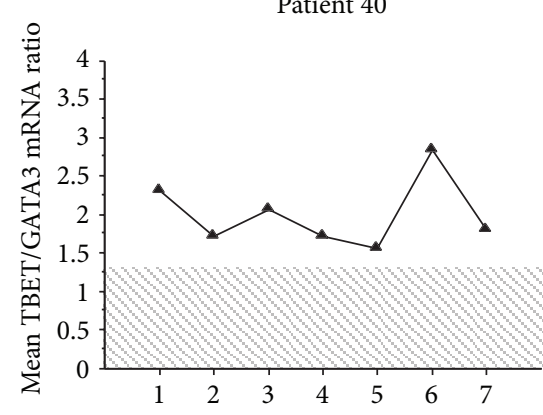

Time from symptom onset (hours)

(b)
Patient 8

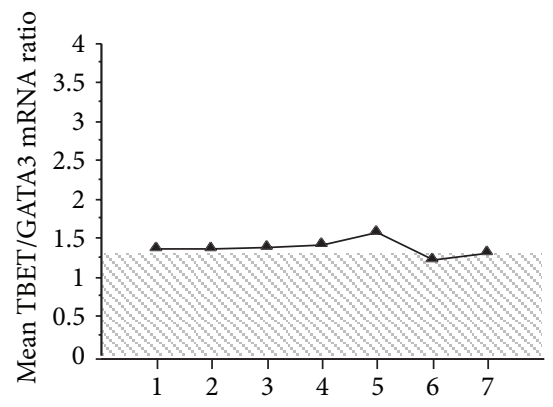

Time from symptom onset (hours)

Patient 25

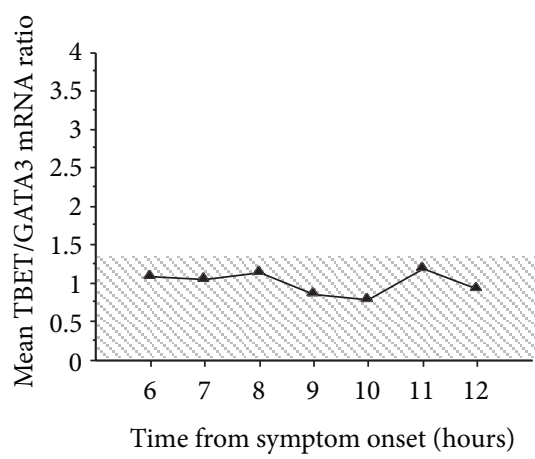

Patient 32

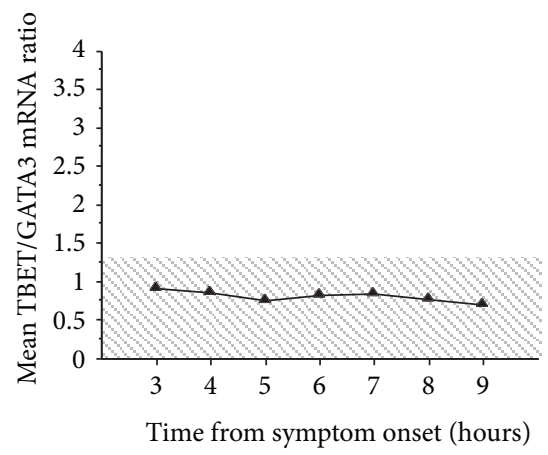

FIgURE 3: Continued. 

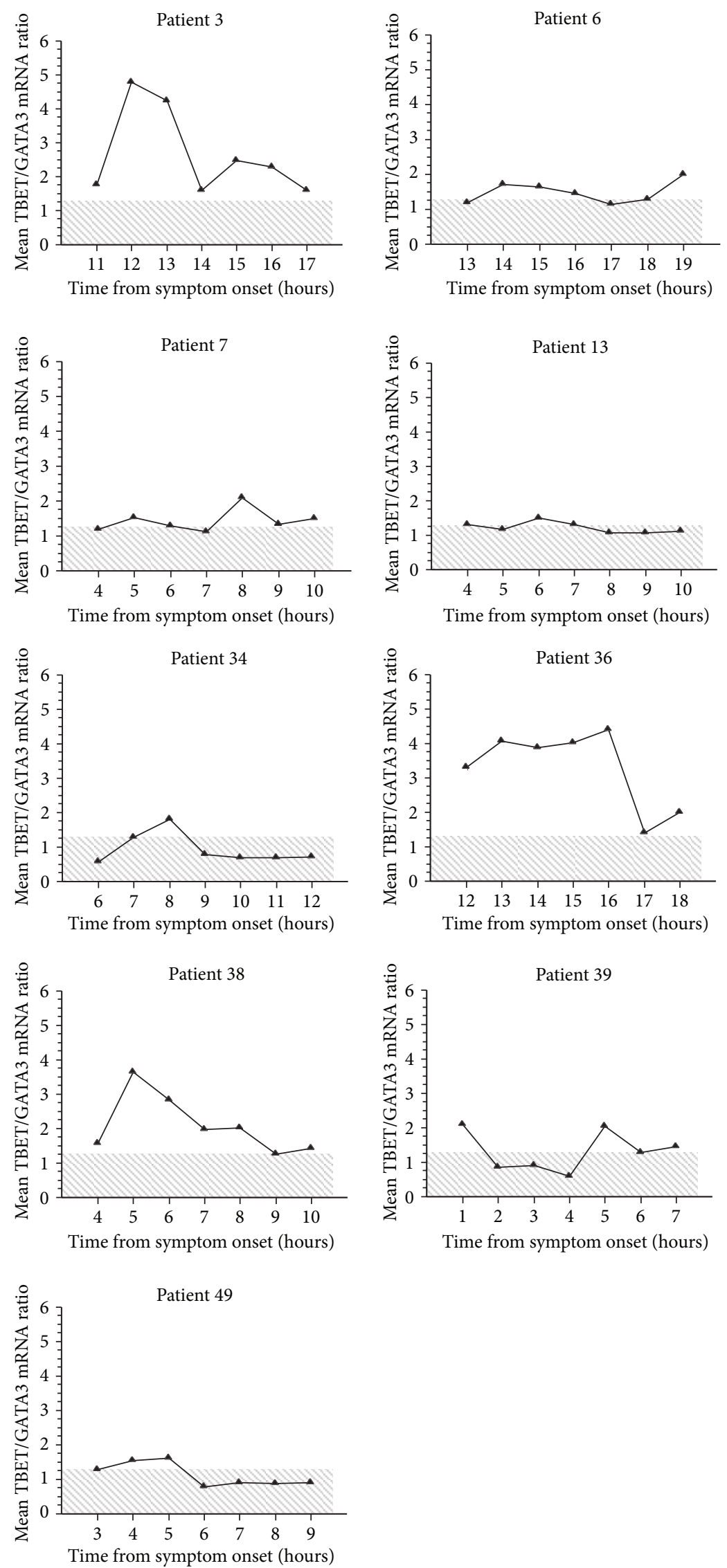

(c)

Figure 3: Continued. 
Patient 2
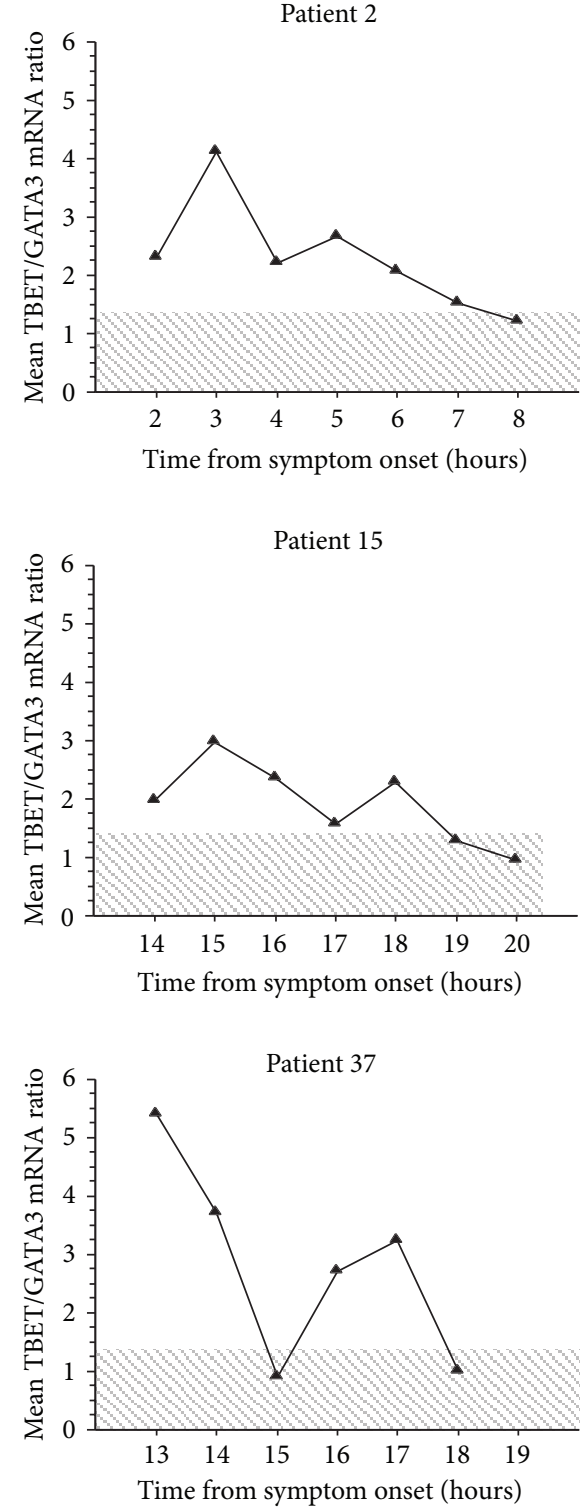

Patient 10



Patient 28



(d)

FIGURE 3: Temporal changes in TBET/GATA3 mRNA ratio in 8 patients with stable angina (a), 11 patients with unstable angina (b), 9 patients with STEMI (c), and 5 patients with NSTEMI (d). The grey zone represents the ratio within the 95th percentile (i.e., $\leq 1.26)$.

differences in the AUC between TBET/GATA3 ratio and initial CK $(0.780 ; 95 \%$ CI 0.700-0.847; $P=0.0142)$, initial cTnT (0.785; 95\% CI $0.705-0.851 ; P=0.0116)$, GATA3 mRNA (0.704; 95\% CI 0.643-0.796; $P=0.0001)$, and TBET mRNA (0.510; 95\% CI $0.422-0.597 ; P<0.0001)$.

This study was the first to demonstrate distinct temporal changes of the TBET/GATA3 mRNA ratios in AMI patients, non-AMI patients, and healthy control subjects. The TBET/ GATA3 mRNA ratio was elevated dramatically in AMI patients and exhibited biphasic $\mathrm{M}$-shaped release kinetics with two distinct peaks. The first blood samples taken from the 14 AMI patients prior to any intervention in the current study also showed the biphasic M-shaped curve. However, the sample size was too small for robust analysis and drawing any conclusion. Therefore, the blood samples taken from the 47 AMI patients prior to any intervention in our previous study were used for analysis by plotting the change of the TBET/GATA3 ratio against the symptom onset. A similar biphasic $\mathrm{M}$-shaped curve was observed. This indicates that the temporal changes in the TBET/GATA 3 mRNA ratio were associated with the development of the pathophysiological processes rather than the effect of therapeutic intervention. Besides, the temporal changes of the TBET/GATA3 ratio in the 89 non-AMI patients recruited in the previous study resembled those in the AMI patients. However, this was not observed in the 19 non-AMI patients recruited in the current study. The difference may be explained by the difference in the proportions of the UA and SA patients. With a much higher 




FIgURE 4: Box-plot of TBET/GATA3 mRNA ratio at $T_{0}$ in healthy subjects $(N=28)$, in patients with stable angina $(N=8)$, unstable angina $(N=11)$, NSTEMI $(N=5)$, and STEMI $(N=9)$. The differences across the groups were significant (Kruskal-Wallis test, $P=0.0006$ ), while the differences between groups are compared using Mann-Whitney $U$ test. The lines inside the boxes denote medians, while the boxes mark the interval between the 25th and 75 th percentiles. The whiskers denote the interval between the 10th and 90th percentiles.

proportion of UA patients ( $80.9 \%$ versus $57.9 \%$ ), the curve shape was much closer to that of AMI patients, but the peaks were less pronounced compared to those of AMI patients.

The biphasic release kinetics of TBET/GATA3 mRNA ratio suggested that significant pathophysiological processes were underway which could reflect a surge in inflammatory and/or immunological activity in ACS. One possible explanation is that the first peak represents the increased inflammatory activity associated with plaque rupture and a distinct Thl mediated response to coronary arterial inflammation in ACS patients [12]. The second peak may reflect the active immunological response to myocardial necrosis. The loss of Th1/Th2 balance in peripheral blood of ACS patients may represent distinct inflammatory cascades mediating the myocardial inflammation after myocardial necrosis which leads to ventricular remodeling [12].

Several studies have shown that $\mathrm{Th} 1 / \mathrm{Th} 2$ imbalance is implicated in the ACS process $[6,9,12,28]$. This imbalance is particularly related to more severe ACS and tends to skew towards Thl predominance. However, the sampling procedures did not specify exact timings and generally related timings to ED arrival rather than symptom onset. The more precise timing of our study allowed a more detailed analysis of the temporal variations of TBET/GATA3 mRNA ratio, which in turn reflected Th1/Th2 balance in ACS patients. TBET/ GATA3 mRNA ratio may act as an alternative measure of Th1/Th2 cytokine profiles as the altered Th1/Th2 cytokine expression in ACS is the result of a shift of TBET and GATA3 profiles. In line with other studies, Th1/Th2 balance was reflected more closely by the TBET/GATA 3 mRNA ratio than the variations of absolute TBET and GATA3 expressions [29].

There were two limitations in this study. Firstly, the number of participants was relatively small. Further studies with



FIgURE 5: Temporal changes in mean TBET/GATA3 mRNA ratio after symptom onset in 47 AMI patients $(\bullet)$ and 89 non-AMI patients (o) recruited in our previous study [14]. Data is presented as mean \pm 1SEM. The grey zone represents the ratio within the 95th percentile (i.e., $\leq 1.26$ ). Significant difference in TBET/GATA3 mRNA ratio was found between AMI and non-AMI patients with $P<0.05$ by using $t$-test $(*)$ or post hoc Bonferroni test $(* *)$.

larger sample sizes are required to investigate the diagnostic and prognostic windows by using the TBET/GATA3 ratio in ACS. These studies should provide statistical confirmation of the optimal cutoff value for the ratio. Secondly, it was not possible to take samples at all the proposed time points which in turn rendered statistical analysis difficult. This study was conducted in a real-life context. Precise study conditions, especially sampling, were curtailed because patients required emergency therapeutic procedures. In order to proceed with statistical analysis, missing data points were filled using a statistical technique described by Snedecor and Cochran [18].

In conclusion, this study has demonstrated that the TBET/GATA3 mRNA ratio was elevated in patients with AMI throughout most of the first 20 hours after onset of acute symptoms. The biphasic $\mathrm{M}$-shaped release kinetics was more likely to reflect pathophysiological changes rather than treatment effects.

\section{Abbreviations}

TBET: T-box expressed in T cells

GATA3: Guanine adenine thymine adenine sequence-binding protein 3

ACS: $\quad$ Acute coronary syndrome

AMI: Acute myocardial infarction

Th: T-helper

SA: Stable angina

UA: Unstable angina

ED: $\quad$ Emergency department

cTnT: $\quad$ Cardiac troponin T

ECG: Electrocardiogram

LBBB: Left branch bundle block

STEMI: ST elevation myocardial infarction

NSTEMI: Non-ST elevation MI

PCI: Percutaneous coronary intervention 
RT-qPCR: Real-time quantitative reverse transcription polymerase chain reaction

GAPDH: Glyceraldehyde 3-phosphate dehydrogenase IQR: Interquartile range.

\section{Acknowledgment}

This study was supported by the Chinese University of Hong Kong Direct Grant for Research Project (Code 2041171).

\section{References}

[1] W.-P. Zheng and R. A. Flavell, "The transcription factor GATA3 is necessary and sufficient for Th2 cytokine gene expression in CD4 T cells," Cell, vol. 89, no. 4, pp. 587-596, 1997.

[2] S. J. Szabo, S. T. Kim, G. L. Costa, X. Zhang, C. G. Fathman, and L. H. Glimcher, "A novel transcription factor, T-bet, directs Th1 lineage commitment," Cell, vol. 100, no. 6, pp. 655-669, 2000.

[3] K. M. Murphy and S. L. Reiner, "The lineage decisions of helper T cells," Nature Reviews Immunology, vol. 2, no. 12, pp. 933-944, 2002.

[4] S. J. Szabo, B. M. Sullivan, S. L. Peng, and L. H. Glimcher, "Molecular mechanisms regulating Th1 immune responses," Annual Review of Immunology, vol. 21, pp. 713-758, 2003.

[5] E. Laurat, B. Poirier, E. Tupin et al., "In vivo downregulation of T helper cell 1 immune responses reduces atherogenesis in apolipoprotein E-knockout mice," Circulation, vol. 104, no. 2, pp. 197202, 2001.

[6] H. Methe, S. Brunner, D. Wiegand, M. Nabauer, J. Koglin, and E. R. Edelman, "Enhanced T-helper-1 lymphocyte activation patterns in acute coronary syndromes," Journal of the American College of Cardiology, vol. 45, no. 12, pp. 1939-1945, 2005.

[7] M. Fisher and E. Folland, "Acute ischemic coronary artery disease and ischemic stroke: similarities and differences," American Journal of Therapeutics, vol. 15, no. 2, pp. 137-149, 2008.

[8] Q.-W. Ji, M. Guo, J.-S. Zheng et al., "Downregulation of T helper cell type 3 in patients with acute coronary syndrome," Archives of Medical Research, vol. 40, no. 4, pp. 285-293, 2009.

[9] H. Soejima, A. Irie, S. Miyamoto et al., "Preference toward a Thelper type 1 response in patients with coronary spastic angina," Circulation, vol. 107, no. 17, pp. 2196-2200, 2003.

[10] G. B. Sajan, T. Z. Qiu, X. Wang, and H.-P. Guo, “T helper cell related interleukins and the angiographic morphology in unstable angina," Cytokine, vol. 30, no. 5, pp. 303-310, 2005.

[11] H. Yamashita, K. Shimada, E. Seki, H. Mokuno, and H. Daida, "Concentrations of interleukins, interferon, and C-reactive protein in stable and unstable angina pectoris," American Journal of Cardiology, vol. 91, no. 2, pp. 133-136, 2003.

[12] X. Cheng, Y.-H. Liao, H. Ge et al., “Th1/Th2 functional imbalance after acute myocardial infarction: coronary arterial inflammation or myocardial inflammation," Journal of Clinical Immunology, vol. 25, no. 3, pp. 246-253, 2005.

[13] A. Adler, Y. Levy, A. Roth, D. Wexler, G. Keren, and J. George, "Functional T-lymphocyte dichotomy in the peripheral blood of patients with unstable angina," International Journal of Cardiovascular Interventions, vol. 7, no. 3, pp. 146-151, 2005.

[14] T. H. Rainer, R. W. Y. Chan, C. A. Graham et al., "Circulating leukocyte TBET and GATA3 mRNA in patients with acute coronary syndrome," International Journal of Cardiology, vol. 156, no. 2, pp. 209-211, 2012.

[15] J. L. Anderson, C. D. Adams, E. M. Antman et al., "ACC/AHA 2007 guidelines for the management of patients with unstable angina/non-ST-Elevation myocardial infarction: a report of the American College of Cardiology/American Heart Association Task Force on Practice Guidelines (Writing Committee to Revise the 2002 Guidelines for the Management of Patients With Unstable Angina/Non-ST-Elevation Myocardial Infarction) developed in collaboration with the American College of Emergency Physicians, the Society for Cardiovascular Angiography and Interventions, and the Society of Thoracic Surgeons endorsed by the American Association of Cardiovascular and Pulmonary Rehabilitation and the Society for Academic Emergency Medicine," Journal of the American College of Cardiology, vol. 50, no. 7, pp. e1-e157, 2007.

[16] K. Thygesen, J. S. Alpert, and H. D. White, "Joint ESC/ACCF/ AHA/WHF task force for the redefinition of myocardial infarction. Universal definition of myocardial infarction2007," Journal of the American College of Cardiology, vol. 50, pp. 2173-2195, 2007.

[17] E. M. Antman, D. T. Anbe, P. W. Armstrong et al., "ACC/AHA guidelines for the management of patients with ST-elevation myocardial infarction: a report of the American College of Cardiology/American Heart Association Task Force on Practice Guidelines (Committee to Revise the 1999 Guidelines for the Management of Patients with Acute Myocardial Infarction)," Circulation, vol. 110, no. 9, pp. e82-e292, 2004.

[18] G. W. Snedecor and W. G. Cochran, Statistical Methods, The Iowa State University Press, Ames, Iowa, USA, 6th edition, 1971.

[19] G. K. Hansson, "Immune mechanisms in atherosclerosis," Arteriosclerosis, Thrombosis, and Vascular Biology, vol. 21, pp. 18761890, 2001.

[20] P. Libby, "Current concepts of the pathogenesis of the acute coronary syndromes," Circulation, vol. 104, no. 3, pp. 365-372, 2001.

[21] G. J. Blake and P. M. Ridker, "Novel clinical markers of vascular wall inflammation," Circulation Research, vol. 89, no. 9, pp. 763771, 2001.

[22] L. Lind, "Circulating markers of inflammation and atherosclerosis," Atherosclerosis, vol. 169, no. 2, pp. 203-214, 2003.

[23] L. M. Biasucci, "CDC/AHA Workshop on Markers of Inflammation and Cardiovascular Disease: application to Clinical and Public Health Practice: clinical use of inflammatory markers in patients with cardiovascular diseases: a background paper," Circulation, vol. 110, no. 25, pp. e560-e567, 2004.

[24] S. J. Szabo, B. M. Sullivan, C. Sternmann, A. R. Satoskar, B. P. Sleckman, and L. H. Glimcher, "Distinct effects of T-bet in Th1 lineage commitment and IFN- $\gamma$ production in CD4 and CD8 T cells," Science, vol. 295, no. 5553, pp. 338-342, 2002.

[25] P. R. Moreno, E. Falk, I. F. Palacios, J. B. Newell, V. Fuster, and J. T. Fallon, "Macrophage infiltration in acute coronary syndromes: implications for plaque rupture," Circulation, vol. 90, no. 2, pp. 775-778, 1994.

[26] E. Lantelme, S. Mantovani, B. Palermo, R. Campanelli, F. Sallusto, and C. Giachino, "Kinetics of GATA-3 gene expression in early polarizing and committed human T cells," Immunology, vol. 102, no. 2, pp. 123-130, 2001.

[27] H. J. Lee, N. Takemoto, H. Kurata et al., "GATA-3 induces T helper cell type 2 (Th2) cytokine expression and chromatin remodeling in committed Th1 cells," Journal of Experimental Medicine, vol. 192, no. 1, pp. 105-115, 2000. 
[28] J. L. Fernandes, R. L. Mamoni, J. L. Orford et al., "Increased Th1 activity in patients with coronary artery disease," Cytokine, vol. 26, no. 3, pp. 131-137, 2004.

[29] H. Chakir, H. Wang, D. E. Lefebvre, J. Webb, and F. W. Scott, "T-bet/GATA-3 ratio as a measure of the Th1/Th2 cytokine profile in mixed cell populations: Predominant role of GATA-3," Journal of Immunological Methods, vol. 278, no. 1-2, pp. 157-169, 2003. 


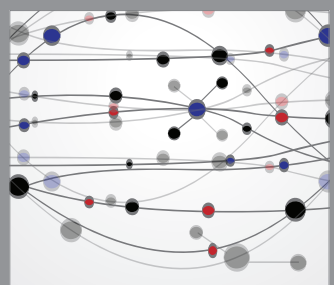

The Scientific World Journal
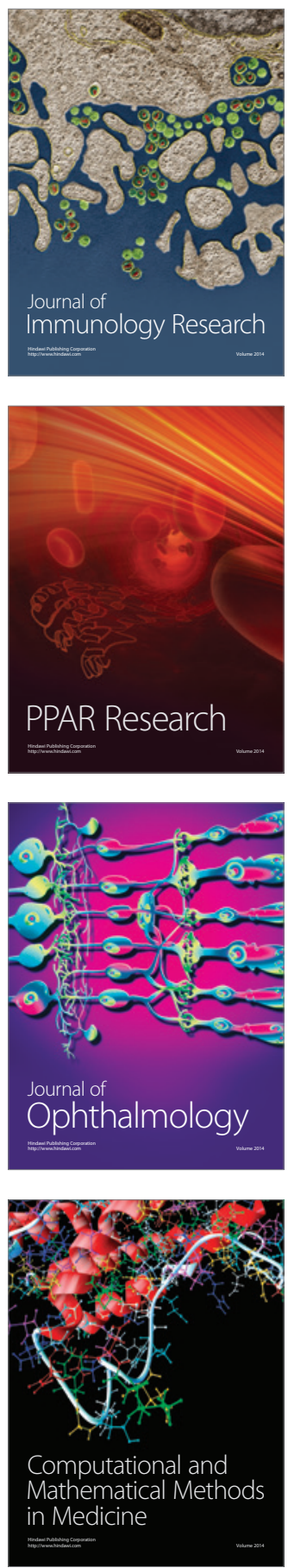

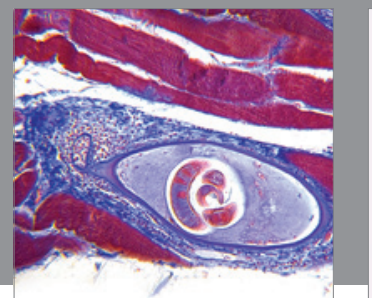

Gastroenterology

Research and Practice


\section{Hindawi}

Submit your manuscripts at

http://www.hindawi.com
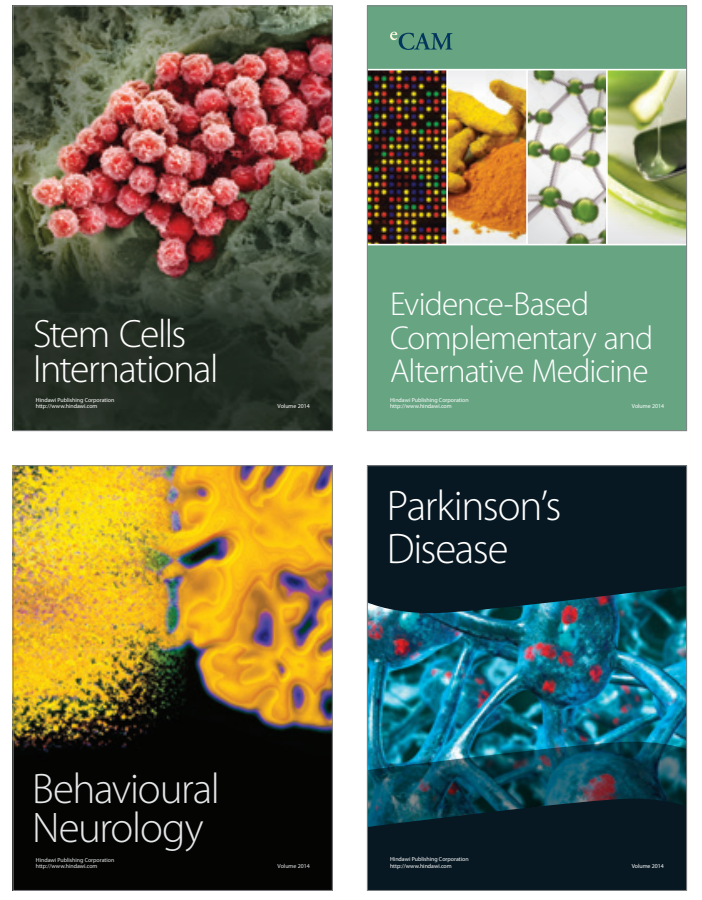
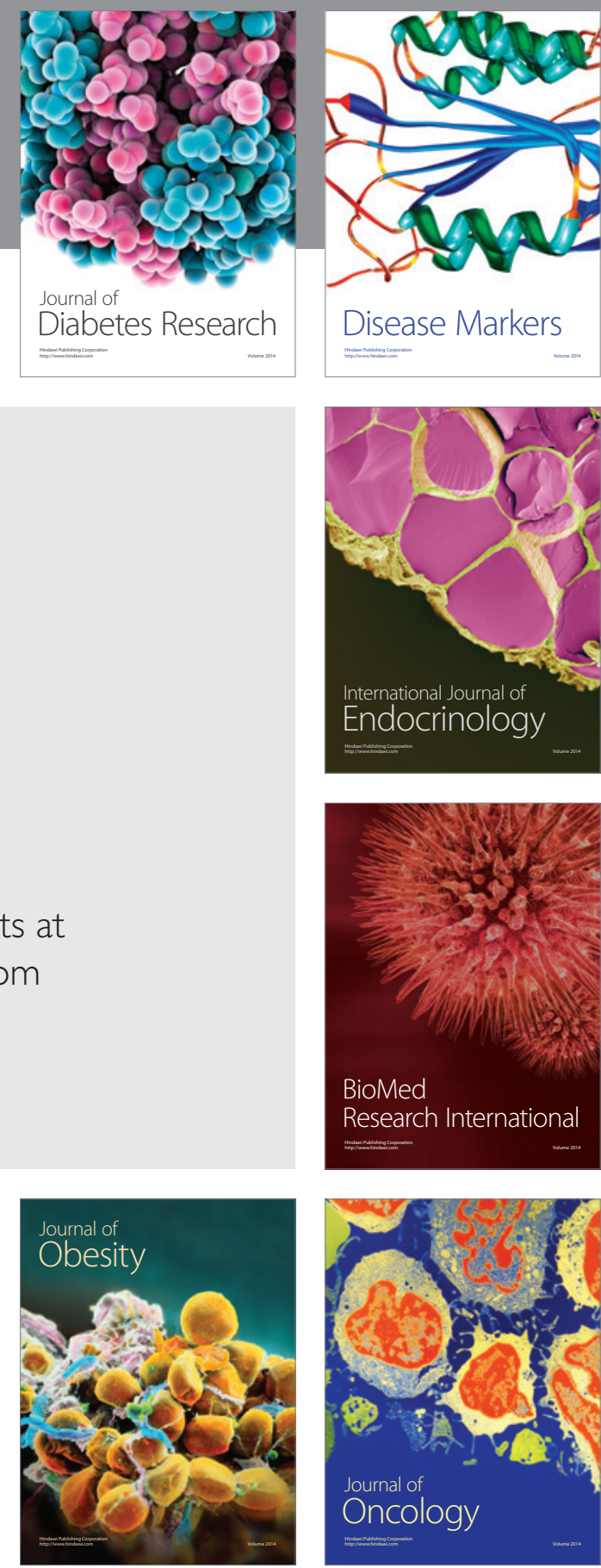

Disease Markers
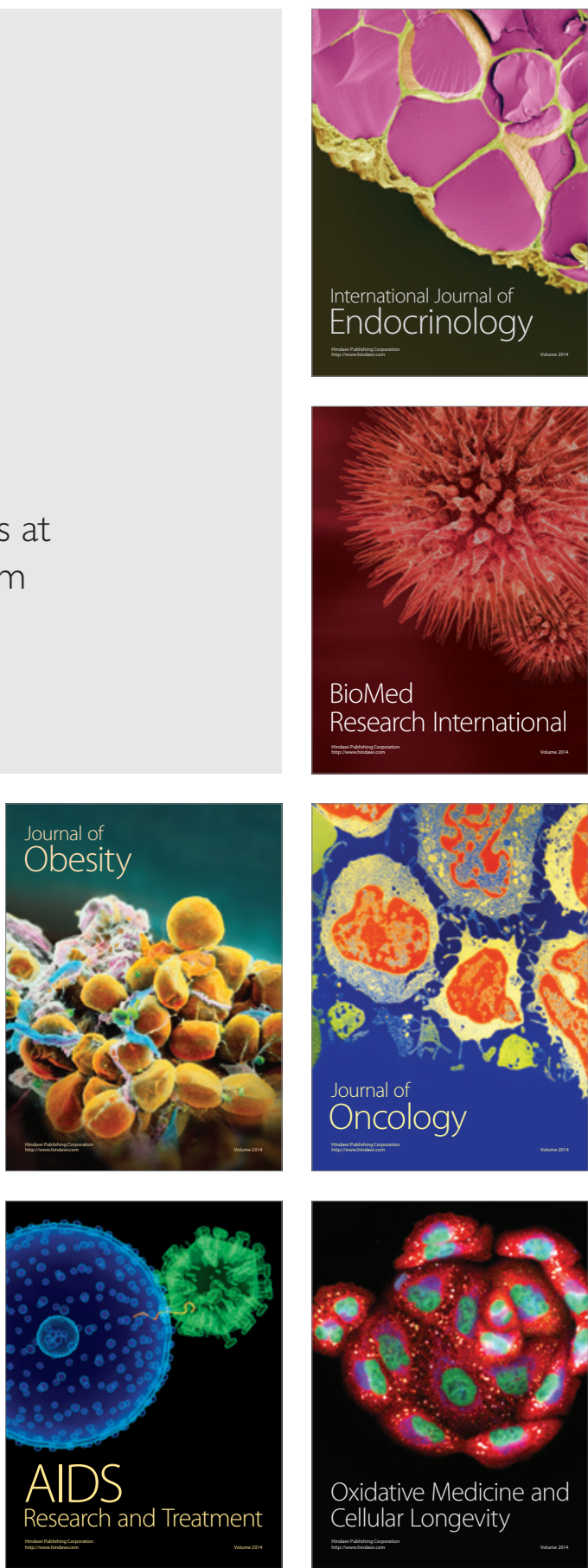